\title{
Prolonged rod dark adaptation in retinitis pigmentosa
}

\author{
KENNETH R. ALEXANDER AND GERALD A. FISHMAN \\ From the Department of Ophthalmology, University of Illinois Eye and Ear Infirmary, Chicago
}

SUMMARY The time course of rod dark adaptation was measured in 12 patients and carriers of retinitis pigmentosa (RP). In contrast to previous studies the rod absolute threshold was determined prior to any exposure to the bleaching light. For seven of the patients and carriers the recovery of rod sensitivity to the prebleach level was prolonged, with the delay ranging from mild to severe. The prolongation appeared to be limited to the late phase of bleaching recovery; the early portion of rod dark adaptation, measured following a weak bleach, was normal. These results suggest a selective impairment in the processes underlying rod dark adaptation in some patients with RP.

Retinitis pigmentosa (RP) is a group of genetic retinal dystrophies with characteristic symptoms and signs that include nightblindness, intraretinal bone-spiculelike pigmentation, narrowing of the retinal vessels, visual field depressions or scotomata, and a reduction in the scotopically, and frequently the photopically, obtained electroretinogram (ERG). ' Genetic types include autosomal dominant, autosomal recessive, and X-linked recessive inheritance.

Studies of psychophysical dark adaptation in RP patients have reported that rod thresholds are often elevated, but that the time course of the recovery of rod sensitivity is normal. ${ }^{2-4}$ Pugh $^{5}$ has argued, however, that the time course of dark adaptation cannot be accurately determined unless the darkadapted rod threshold is known prior to the bleach. By first determining prebleach thresholds in normal persons he has shown that the time course of human psychophysical dark adaptation is somewhat longer than previously estimated. A similar consideration applies to patients with RP. The elevated rod thresholds observed in RP patients during dark adaptation might be due at least partially to a delayed recovery of rod system sensitivity as well as to a decreased sensitivity of the rod system per se. Consequently we have measured bleaching recovery functions in nine patients with RP, and in three carriers of $X$-linked recessive $R P$, after first measuring the prebleach rod sensitivity to determine whether the recovery of rod sensitivity proceeds at a normal rate.

Correspondence to Kenneth R. Alcxander, PhD, University of Illinois Eyc and Ear Infirmary, 1855 W Taylor Strect, Chicago, IL 60612, USA.
Dark adaptation ultimately depends on the regeneration of bleached photopigments. ${ }^{6-8}$ However, additional processes besides the regeneration of bleached rhodopsin contribute to the threshold elevations observed during rod dark adaptation, particularly during the early phase, which can usually be measured only after a weak bleach. ${ }^{8-10}$ Therefore we have measured dark adaptation in two RP patients after a weak bleach to determine whether there is a selective alteration of different phases of rod dark adaptation.

\section{Materials and methods}

Nine RP patients and three carriers of $X$-linked recessive RP participated in the study. Data are presented for five of the RP patients and two of the carriers who had an abnormal rate of dark adaptation. Their ophthalmological characteristics are given in Table 1. Four patients (two with autosomal dominant and two with X-linked recessive $R P$ ) and one additional $\mathrm{X}$-linked recessive carrier had normal dark adaptation curves. Their ocular characteristics did not differ in any systematic way from the group of patients with abnormal dark adaptation. Six persons who had a normal ocular examination and who were comparable in age to the RP patients and carriers served as controls. During the experiments the subjects' pupils were dilated and accommodation was paralysed by the instillation of $10 \%$ phenylephrine and $1 \%$ cyclopentolate drops. No optical corrections were used.

Threshold testing was carried out with a Tübinger perimeter. The circular test stimulus (TS) was $104^{\prime}$ in diameter and was either of a long wavelength (Oculus 
Table 1 Patient characteristics

\begin{tabular}{|c|c|c|c|c|c|c|c|c|c|c|}
\hline \multirow{2}{*}{$\begin{array}{l}\text { Patient } \\
\text { number }\end{array}$} & \multirow{2}{*}{$\begin{array}{l}\text { Age. } \\
\text { years }\end{array}$} & \multirow[t]{2}{*}{ Sex } & \multirow[t]{2}{*}{ Genetic type } & \multirow{2}{*}{$\begin{array}{l}\text { Visual } \\
\text { acuity }\end{array}$} & \multirow{2}{*}{$\begin{array}{l}\text { Field } \\
\text { efficiency* }\end{array}$} & \multicolumn{5}{|c|}{ Electroretinogram $\ddagger$} \\
\hline & & & & & & $\begin{array}{l}\text { Photopic } \\
\text { white }\end{array}$ & $\begin{array}{l}\text { Scotopic } \\
\text { white }\end{array}$ & $\begin{array}{l}\text { Scotopic } \\
\text { blue }\end{array}$ & $\begin{array}{l}\text { Flicker } \\
\text { blue }\end{array}$ & $\begin{array}{l}\text { Flicker } \\
\text { red }\end{array}$ \\
\hline 1 & 49 & $\mathbf{F}$ & Autos. dom. & $20 / 20^{-2}$ & 53 & $80(36)$ & $150(54)$ & ND & $12(84)$ & $14(44)$ \\
\hline 2 & 28 & F & X-1. carricr & $20 / 30^{-1}$ & 23 & ND & $50(60)$ & ND & $8(97)$ & ND \\
\hline 3 & 35 & $M$ & Autos. dom. & $20 / 20^{-2}$ & 45 & $100(38)$ & $150(44)$ & ND & $12(78)$ & $15(45)$ \\
\hline 4 & 17 & $\mathbf{F}$ & Autos. dom. & $20 / 40^{-2}$ & 45 & ND & $40(64)$ & ND & ND & ND \\
\hline 5 & 28 & $\mathrm{~F}$ & Autos. dom. & $20 / 25$ & $100(50) \dagger$ & $90(26)$ & $370(48)$ & $190(70)$ & $32(82)$ & $13(41)$ \\
\hline 6 & 29 & $F$ & Autos. dom. & $20 / 30$ & 54 & $90(30)$ & $390(60)$ & $190(80)$ & $34(77)$ & $14(43)$ \\
\hline 7 & 48 & $\mathrm{~F}$ & X-1. carricr & $20 / 20$ & 76 & $40(36)$ & $170(60)$ & $60(80)$ & $14(103)$ & $6(48)$ \\
\hline & & & & & & $\geqslant 100(\leqslant 34)$ & $\geqslant 400(\leqslant 56)$ & $\geqslant 190(\leqslant 92)$ & $\geqslant 34(\leqslant 89)$ & $\geqslant 14(\leqslant 43)$ \\
\hline
\end{tabular}

${ }^{*}$ Obtained by measuring the angular extent of visual ficld in degrees from the central fixation point along cach of cight meridians, using the Goldmann 4-c-II target, totalling and dividing by $5 .{ }^{11}$

tObtained by using the Goldmann 2-c-II target.

$\ddagger$ The first number in each column refers to ERG amplitude in $\mu \mathrm{V}$; the numbers in parentheses indicate implicit times in ms; ND means non-detectable. Numbers at the bottom of cach column give limits of normal values ( 2 SD from the mean).

glass filter, bandpass $>620 \mathrm{~nm}$ ) or middle wavelength (Oculus interference filter, $\lambda_{\max }=500 \mathrm{~nm}$ ). The TS was presented for $500 \mathrm{~ms}$ against the perimeter diffusing surface at a number of retinal locations relative to a $30^{\prime}$ diameter dim red fixation target. For foveal testing fixation was provided by a diamond-shaped pattern of four red dots, $10^{\prime}$ in diameter, separated by $2^{\circ}$. For the weak bleach experiments, the Tübinger fixation target was replaced by a dim red cross provided by a small projector mounted on the side of the perimeter bowl. The luminance of the TS was changed in $0.1 \log$ unit steps by a series of neutral density filters. Stimulus luminances were calibrated by a Spectra Spotmeter, and calculation of the photopic and scotopic values was based on the nominal colour temperature of the tungsten source, the spectral transmission of the filters (as determined by a Carey spectrophotometer), and the photopic and scotopic luminosity functions.

Prior to the experimental session any exposure to bright lights was avoided, and the subject was darkadapted for 45 to $60 \mathrm{~min}$. The subject's absolute thresholds for the long wavelength $(R)$ and middle wavelength (BG) TS were then measured across the horizontal and vertical meridians of the visual field, both to obtain a visual sensitivity profile and to determine whether rods or cones mediated the absolute threshold. ${ }^{12}$ Threshold was defined as the median of three repetitions of an increasing series of TS luminances (ascending method of limits).

Immediately following the threshold profile measurements, which typically required $1 \mathrm{~h}$, two retinal locations were chosen for dark adaptation testing such that rod sensitivity was most nearly optimal for that patient and such that there was minimal variation in rod sensitivity across that retinal region. To make certain that the absolute threshold had been reached the thresholds at these locations were remeasured just prior to the bleaching recovery procedure. The subject's eye was then exposed for 2 min to a Ganzfeld light of $3.6 \mathrm{log} \mathrm{cd} \mathrm{m}^{-2}$, provided by a Feldman Adaptometer (American Optical Co.), placed beside the perimeter. The Ganzfeld light bleached approximately $85 \%$ of the rhodopsin in the normal eye. ${ }^{79}$ Dark adaptation was tested at the two chosen retinal locations by alternating the position of the BG test target, so that dark adaptation curves at the two retinal loci were measured in a single bleaching recovery session. Threshold was obtained by an ascending method of limits, with each datum point representing the end point of a single series of increasing TS luminance.

Dark adaptation testing was continued as long as was practical for patient tolerance, given the extended nature of the entire series of tests. In cases of prolonged dark adaptation the threshold may not have reached the prebleach level by the end of the session. However, enough of the dark adaptation curve was obtained to determine the time constant of recovery.

The earliest part of rod dark adaptation cannot be measured after a moderate bleaching light, since cones determine the threshold immediately following the bleach. However, the early phase of rod dark adaptation can be observed if the bleaching light is relatively weak. We measured rod dark adaptation following a weak bleach in two patients with RP. Each subject first was dark-adapted as described above, then the absolute threshold was measured at the location to be tested during bleaching recovery. For the weak bleach the $11^{\circ}$ white field in the Tübinger fixation channel was presented for $30 \mathrm{~s}$ at a luminance $2.4 \log \mathrm{cd} \mathrm{m}^{-2}(5 \cdot 7 \mathrm{log} \operatorname{scot} \mathrm{td} \mathrm{sec}$, assuming a pupil diameter of $8 \mathrm{~mm}$ ). This light was sufficient to bleach approximately $6 \%$ of the rhodopsin in the normal eye in the region exposed to the light. ${ }^{9}$ During the bleaching exposure, the subject fixated on the auxiliary 
fixation target by turning the eye while maintaining a forward head position in the chinrest. Dark adaptation was measured at a single retinal locus, as determined by the placement of the auxiliary fixation target, with the TS presented in the centre of the region formerly occupied by the bleach. Thresholds were measured as described previously. To avoid prolonged lateral gaze the eccentric fixation target was replaced by the normal fixation target, and the TS was moved to the appropriate retinal position after approximately $15 \mathrm{~min}$ of the experimental session had passed. No change in the threshold was noted as a result of this alteration.

\section{Results}

To determine the normal course of rod dark adaptation under our testing conditions we measured 15 dark adaptation curves at seven different retinal loci in our six normal subjects, after first finding the prebleach rod absolute threshold at each retinal location. The locations tested were $10^{\circ}$ superior, $20^{\circ}$ superior, $20^{\circ}$ temporal, $30^{\circ}$ superior, $30^{\circ}$ temporal, $45^{\circ}$ superior, and $45^{\circ}$ temporal to the fovea, corresponding to the locations that were tested in the RP patients and carriers. Although there was some variation in rod absolute thresholds throughout the normal visual field, as reported previously, ${ }^{12}$ the time constants of the rod dark adaptation curves were similar at the various retinal loci, which was in agreement with previous studies. ${ }^{13}$ The mean time constant for the 15 dark adaptation curves was $8.1 \mathrm{~min}(\mathrm{SD}=1.5 \mathrm{~min})$, which is comparable to that found by Pugh ${ }^{5}$ for a similar magnitude of bleach. Therefore our results for the normal observers are consistent with those of previous studies.

Of the $12 \mathrm{RP}$ patients and carriers who were tested five RP patients and two carriers showed an abnormally prolonged period of rod dark adaptation following the Ganzfeld bleach in comparison with the normal subjects. For each individual similar results were obtained at the two retinal loci that were tested. To simplify the presentation of data only one dark adaptation curve is shown for each individual.

In the following figures the prebleach rod absolute threshold value at each tested location was set to zero, and all thresholds measured during dark adaptation were plotted relative to this. (The actual prebleach threshold values for the RP patients and carriers are shown in separate figures.) The normalisation was done to facilitate a visual comparison between the rates of rod dark adaptation for the RP patients and normal persons and has no effect on the quantitative analysis. Since the curves have been plotted with respect to rod thresholds, which may be elevated in the RP patients, comparisons cannot be made between the values of the cone thresholds in these RP patients and normal persons.

One RP patient (no. 1) and one X-linked recessive carrier (no. 2) showed a marked prolongation of rod dark adaptation, based on the length of time necessary to return to the prebleach level. For patient 1 the absolute threshold profiles for the $R$ and $B G$ test targets across the vertical meridian are shown in Fig. 1. Since thresholds are plotted in photopic units, cone-mediated thresholds for the R and BG targets are indicated by coincident data points, while a vertical separation of $2.6 \mathrm{log}$ units between the data points means that the thresholds are rod-mediated. For intermediate vertical separations between the data points the BG TS is detected by rods, while the $\mathrm{R}$ TS is detected by cones. ${ }^{12}$ The shaded regions represent the range of threshold settings for the RBG TS for five normal observers. Near the fovea the thresholds for patient 1 were elevated and conemediated; there was a midperipheral ring scotoma; and then thresholds returned toward normal in the far periphery and were rod-mediated. The arrow on the abscissa indicates the retinal locus for the dark adaptation curve reported for this patient. The absolute threshold for the BG TS at this locus represents the prebleach baseline to which the dark adaptation curve should return.

A dark adaptation curve for this patient and curves for three of the normal observers at a corresponding

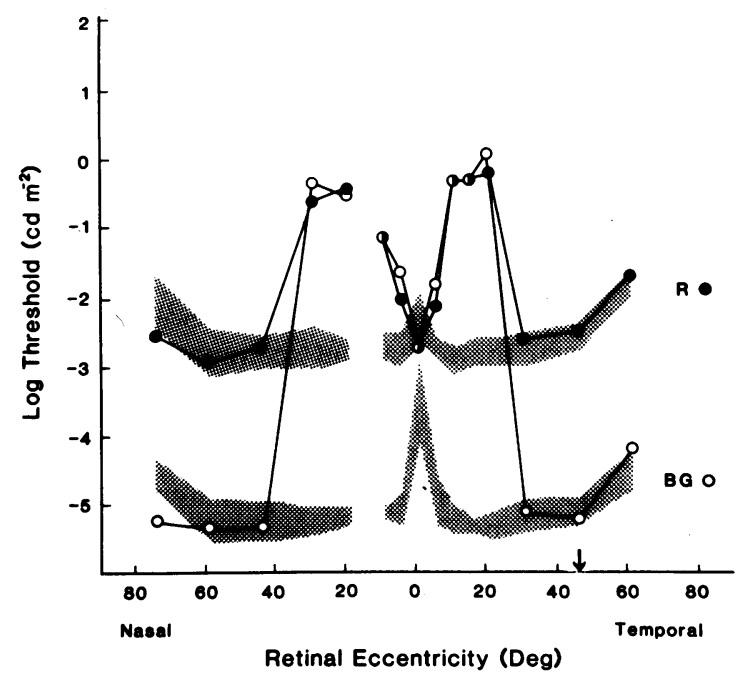

Fig. 1 Absolute thresholds for patient I across the vertical meridian of the visual field, for a middle-wavelength (open circles) and long-wavelength (filled circles) test flash. Shaded regions represent the range of absolute thresholds for the middle-and long-wavelength test flashes for 5 normal subjects. The arrow represents the retinal locus for the dark adaptation curve presented for patient 1 . 
Fig. 2 Thresholds for a middlewavelength test flash during dark adaptation following 2 min exposure to a moderate bleaching light for patient 1 (filled circles) and for three normal subjects (open symbols) at a retinal eccentricity of $45^{\circ}$. Thresholds for all observers are plotted as elevations relative to the respective prebleach absolute thresholds (dashed line). Solid lines represent best-fitting exponentials (fitted to the grouped data for normals), with time constants of 8.5 min (normal persons) and $101.6 \mathrm{~min}$ (patient 1).

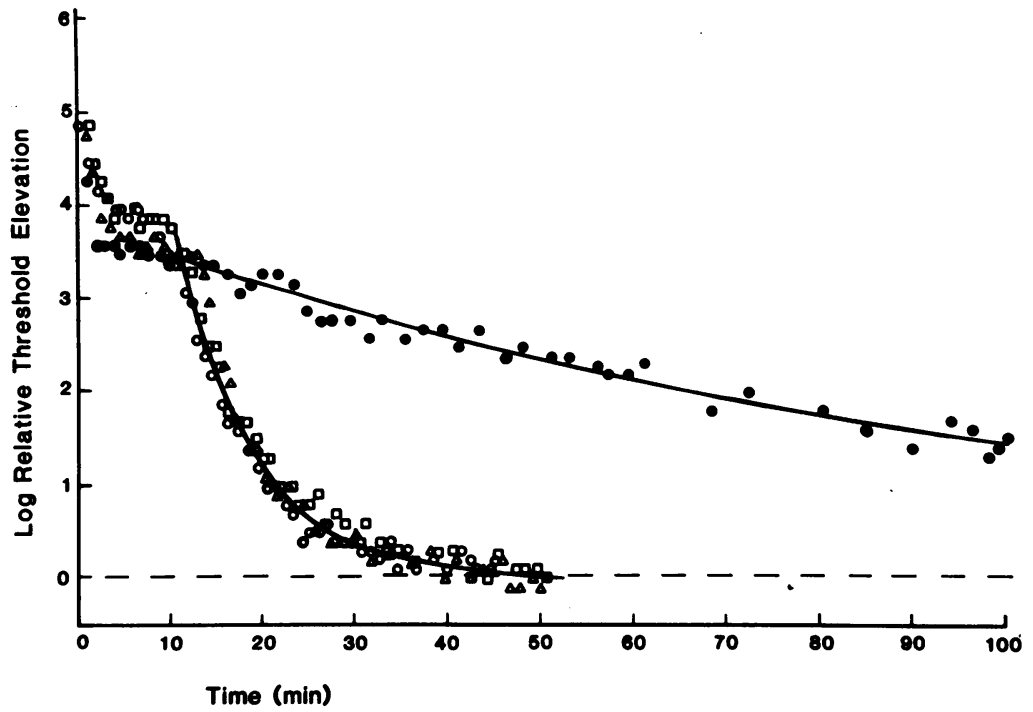

retinal eccentricity are shown in Fig. 2. The dashed line represents the normalised prebleach absolute threshold for each observer, and all thresholds are plotted as elevations relative to this value. The solid lines are best-fitting exponentials fitted to the rod portions of the dark adaptation curves. The mean time constant for the three normal observers was 8.5 $\mathrm{min}$. The time course of dark adaptation for patient 1 was clearly prolonged $(\tau=101.6 \mathrm{~min})$, and the threshold did not reach the baseline even after 100 min in the dark, though it is apparent that the threshold continued to decline throughout the session. A similarly prolonged rod dark adaptation curve was obtained at $30^{\circ}$ in the superior retina of this patient ( $\tau=103.4 \mathrm{~min})$.

A greatly prolonged rod dark adaptation curve was also obtained for patient 2 , an X-linked recessive carrier. Her threshold profile across the vertical meridian (Fig. 3) showed nearly normal BG thresholds near the fovea, but scotomata and field depressions in the mid- to far periphery. One dark adaptation curve, tested at the location shown by the arrow, is presented in Fig. 4. The data from three normal persons at a corresponding retinal eccentricity are also shown, and the solid line through the open symbols represents the best-fitting exponential, with a time constant of $8.8 \mathrm{~min}$.

For patient 2 the dark adaptation curve did not show a marked rod-cone break. However, by measuring thresholds for the $R$ as well as the BG TS during dark adaptation we established that thresholds during the first 7 minutes were cone-determined, whereas all subsequent thresholds were roddetermined. It is apparent that the rod portion of dark adaptation proceeded at two different rates of recovery, with an abrupt transition at approximately $13 \mathrm{~min}$. The exponential that was fitted to the data for patient $2(\tau=121.8 \mathrm{~min})$ was begun at $13 \mathrm{~min}$. (A similar biphasic rod recovery curve was obtained at the second retinal location that was tested in this patient.) A possible explanation for the dual nature of the rod recovery curve will be presented in the 'Discussion.'

Two patients had a moderately prolonged time course of rod dark adaptation. The threshold profile across the vertical meridian for patient 3 (Fig. 5) showed nearly normal BG thresholds near the fovea but a loss of sensitivity toward the retinal periphery.

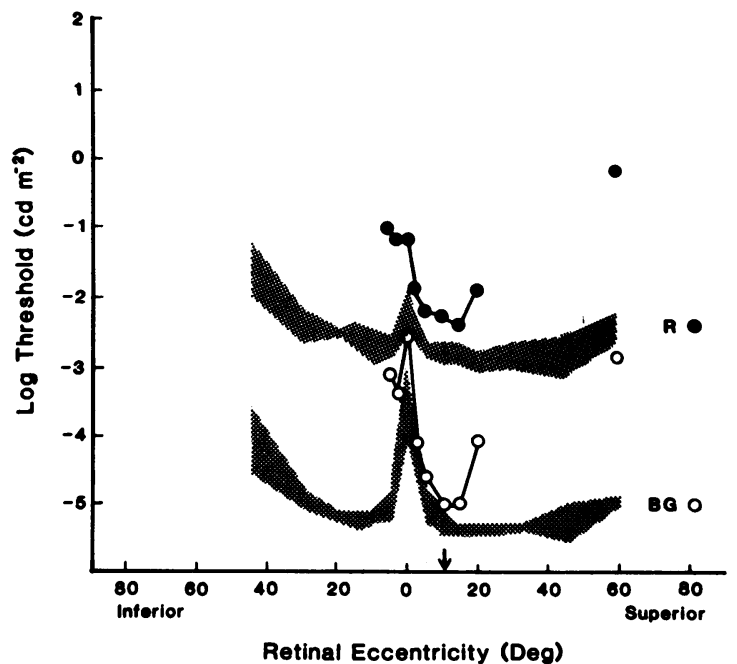

Fig. 3 Absolute thresholds for patient 2 across the vertical meridian of the visual field (other details as in Fig. 1). 


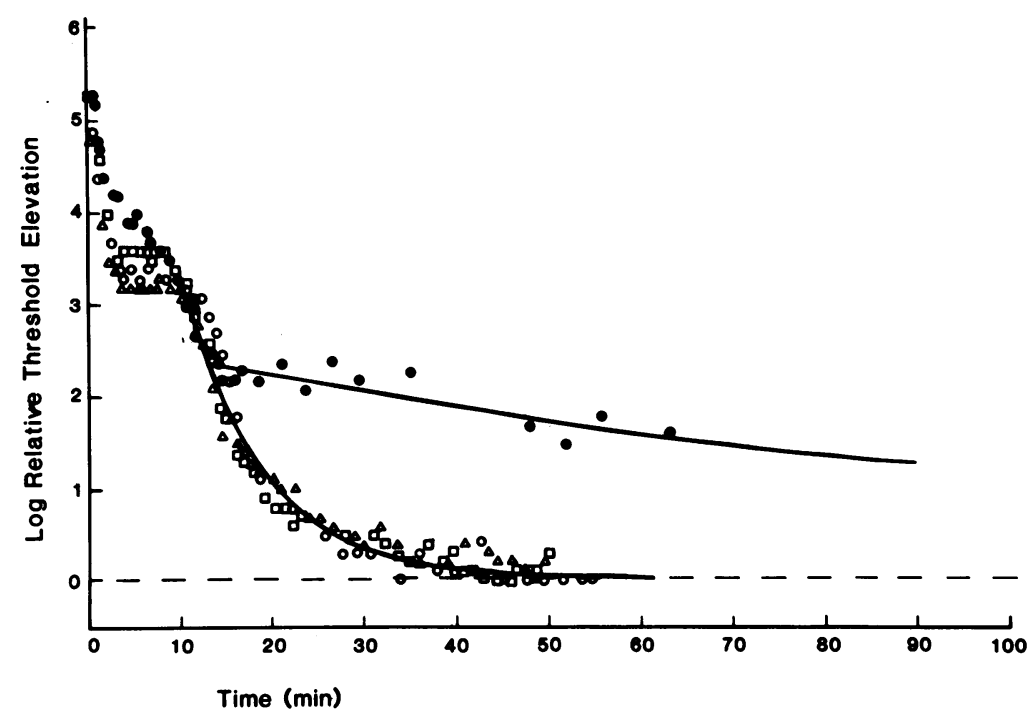

Fig. 4 Thresholds during dark adaptation for patient 2 (filled circles) and three normal subjects (open symbols) at a retinal eccentricity of $10^{\circ}$. The solid lines represent best-fitting exponentials with time constants of $8.8 \mathrm{~min}$ (grouped normals) and $121.8 \mathrm{~min}$ (patient 2).

The threshold profile across the horizontal meridian for patient 4 (Fig. 6) was approximately similar in shape to that for patĩent 3 , in that peripheral thresholds were higher than those near the fovea. However, thresholds for patient 4 were considerably more elevated than those for patient 3. Although patients 3 and 4 are genetically unrelated, and although their threshold profiles showed different degrees of sensitivity loss, their dark adaptation curves were quite similar and have been plotted together in Fig. 7 along with three normal dark adaptation curves at this eccentricity, replotted from

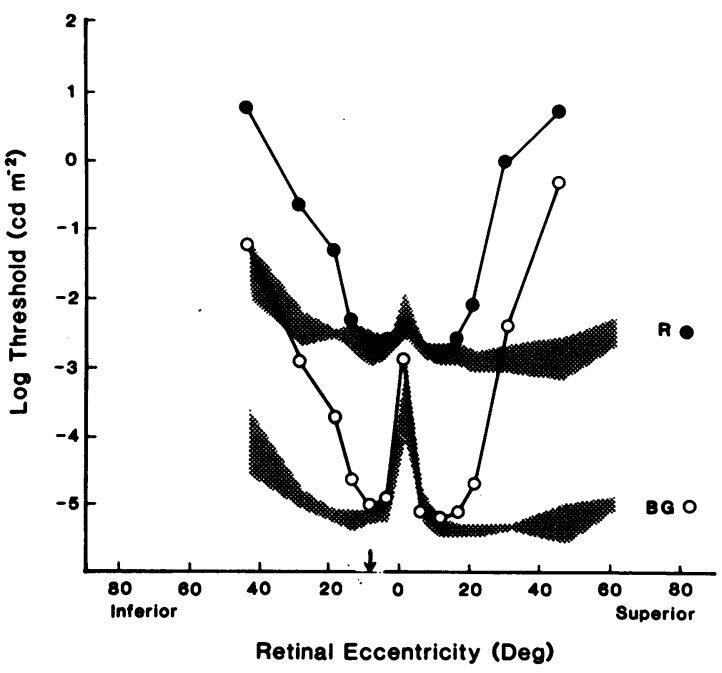

Fig. 5 Absolute thresholds for patient 3 across the vertical meridian of the visual field (other details as in Fig. 1).
Fig. 4. Both RP patients showed a moderately slow return to the baseline threshold $(\tau=19.9 \mathrm{~min}$, grouped data). Similarly prolonged rod dark adaptation curves were obtained at the two other retinal loci that were tested in these two patients.

Three additional patients showed slightly prolonged rod dark adaptation curves, with time constants that were greater than 2 SD from the mean time constant for the normals. The threshold profiles for two of the RP patients, who are sisters, are shown in Figs. 8 and 9. The threshold profiles for both were quite similar, with midperipheral threshold elevations

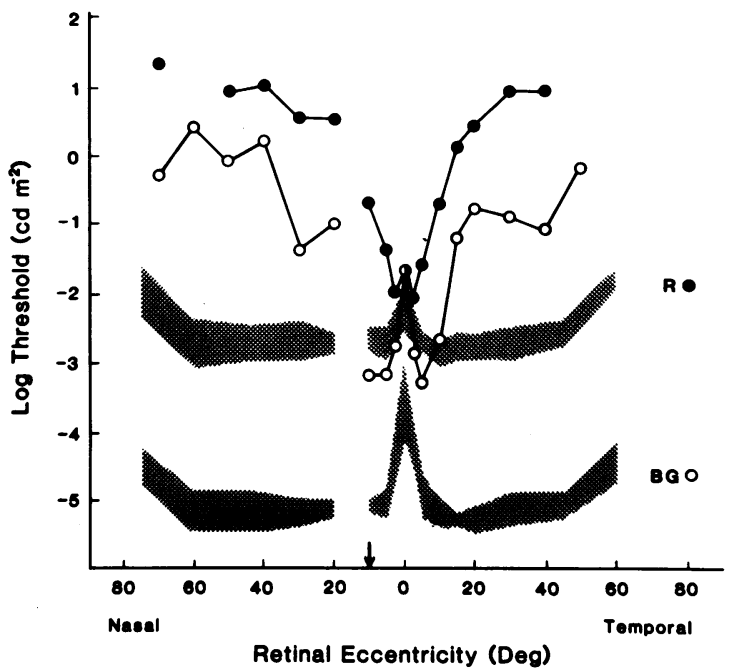

Fig. 6 Absolute thresholds for patient 4 across the horizontal meridian of the visual field (other details as in Fig. I). 
Fig. 7 Thresholds during dark adaptation for patients 3 (filled circles) and 4 (filled triangles) at a retinal eccentricity of $10^{\circ}$. The line through the filled symbols represents the best-fitting exponential, fitted to the grouped data for patients 3 and 4 , with a time constant of $19.9 \mathrm{~min}$. The open symbols represent dark adaptation curves for three normal observers at this eccentricity, replotted from Fig. 4.

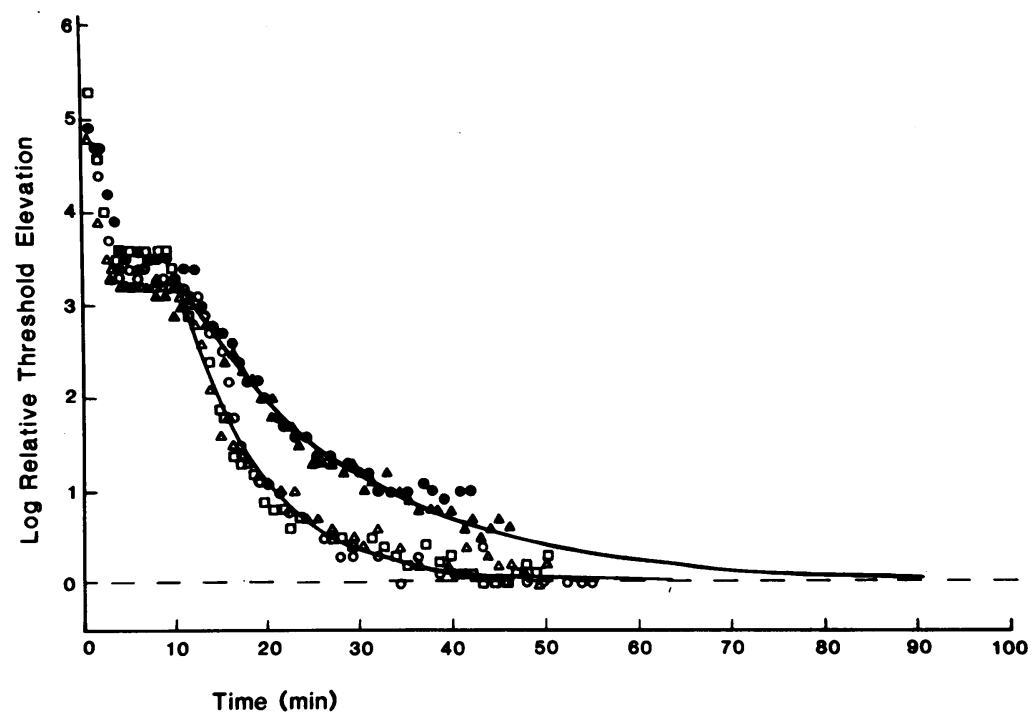

and near normal rod-mediated absolute thresholds in the far periphery. Dark adaptation curves were also quite similar for the two and have been plotted together in Fig. 10, along with three normal dark adaptation curves at this eccentricity, replotted from Fig. 2. Both patients showed a mild prolongation of the return of rod sensitivity $(\tau=11.4 \mathrm{~min}$, grouped data). A mildly prolonged dark adaptation curve $(\tau=$ $13.5 \mathrm{~min}$ ) was also obtained in a third individual (patient 7), a carrier of X-linked RP (Table 1).

Two additional patients with autosomal dominant RP, two with X-linked recessive RP, and one carrier

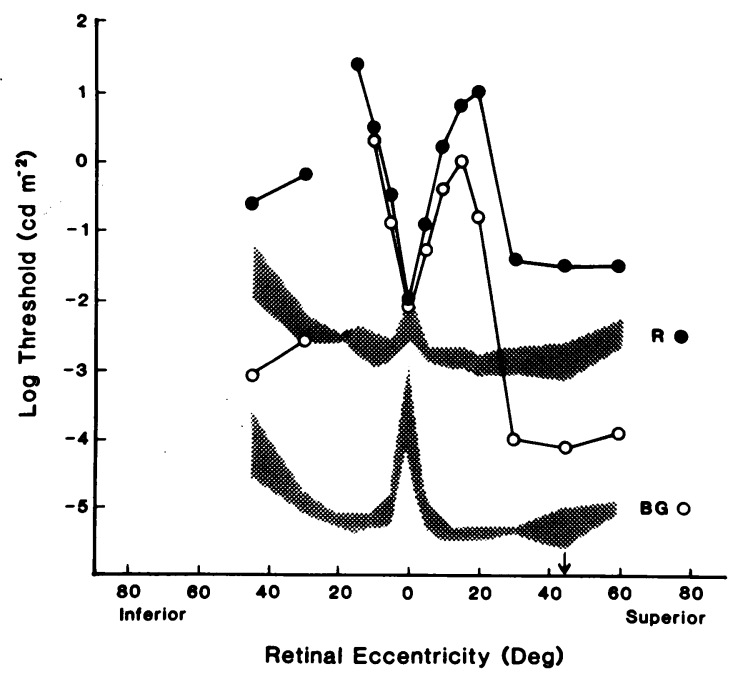

Fig. 8 Absolute thresholds for patient 5 across the horizontal meridian of the visual field (other details as in Fig. I). of X-linked recessive RP had dark adaptation curves with time constants $(7 \cdot 6$ to $10 \cdot 1 \mathrm{~min})$ that fell within $\pm 2 \mathrm{SD}$ of the mean time constant for the normal subjects. All had field defects ranging from mild to severe and had mild to severe reductions in cone and rod ERG amplitudes. There were no apparent systematic differences in ocular characteristics between those individuals showing prolonged dark adaptation and those with normal bleaching recovery.

The recovery of rod sensitivity following exposure to a weak bleaching light is shown for two patients (1 and 3 ) in Figs. 11 and 12. These two patients had

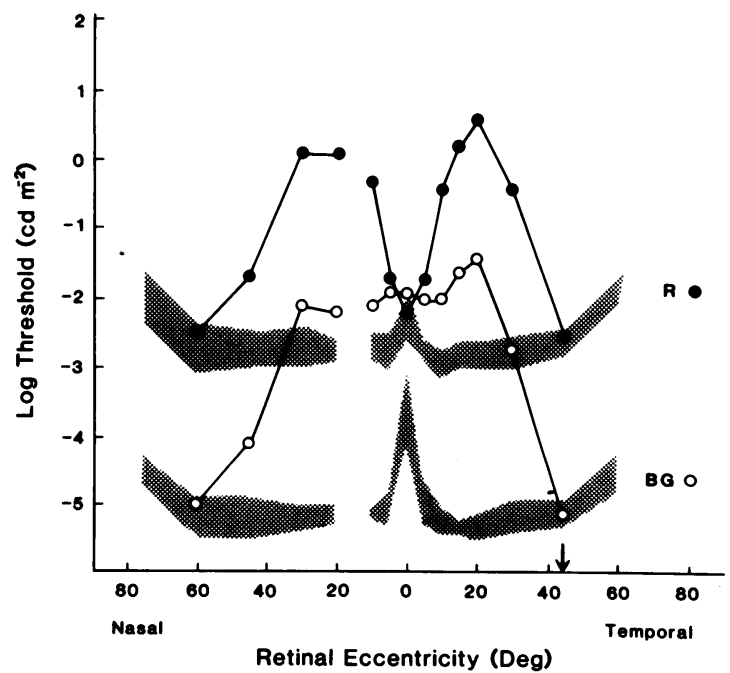

Fig. 9 Absolute thresholds for patient 6 across the horizontal meridian of the visual field (other details as in Fig. 1). 


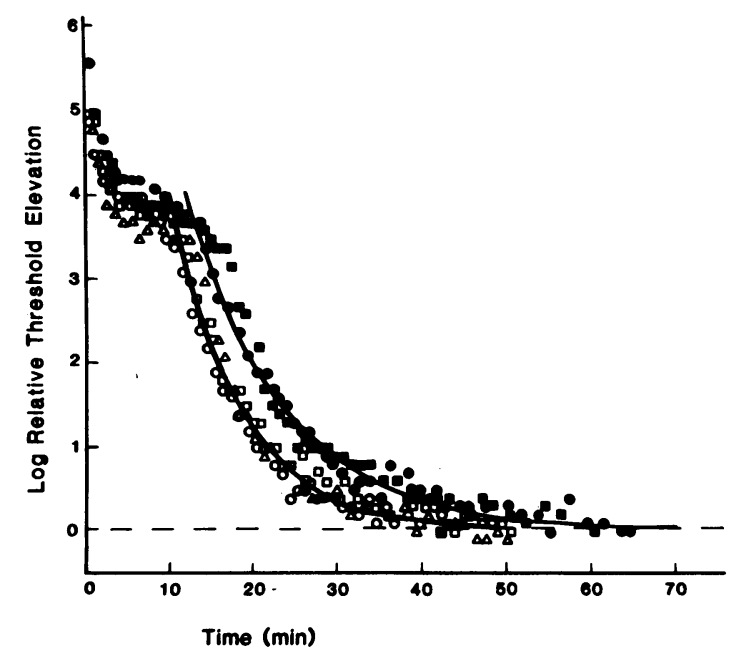

Fig. 10 Thresholds during dark adaptation for patients 5 (filled circles) and 6 (filled squares) at a retinal eccentricity of $45^{\circ}$. The line through the filled symbols represents the bestfitting exponential, fitted to the grouped data for patients 5 and 6 , with a time constant of $11.4 \mathrm{~min}$. The open symbols represent dark adaptation curves for three normal observers at this eccentricity, replotted from Fig. 2.

shown prolonged rod recovery after the moderate bleaching light. Open circles in Figs. 11 and 12 represent dark adaptation for a normal observer; the solid line through the open symbols is the predicted threshold curve based on the regeneration of rhodopsin ( $\tau=6 \mathrm{~min})$. " For the normal observer the thresholds during the initial portion of dark adaptation are considerably higher than predicted from the concentration of bleached rhodopsin. Possible explanations for this discrepancy have been suggested by other workers and will be presented in the Discussion. However, the thresholds during the later phase of bleaching recovery conformed well to the values predicted on the basis of rhodopsin regeneration, as found by previous workers. ${ }^{9}$

For the RP patients the initial portion of the dark adaptation curve following the weak bleach corresponded to the result for the normal observer, suggesting that this earliest part of rod dark adaptation is normal in these two patients. However, near the point where the thresholds apparently begin to be determined by rhodopsin in the normal observer the dark adaptation curves for the RP patients showed a decreased rate of recovery. For the RP patients the best-fit exponentials $(\tau=52.7 \mathrm{~min}$, patient $1 ; 13 \mathrm{~min}$, patient 3) were begun at the point where thresholds diverged from normal. As with the moderate bleach, the recovery of rod sensitivity following a weak bleach took a considerably longer time than normal in these RP patients.

\section{Discussion}

Previous investigators have reported that the time course of rod dark adaptation in RP is normal. ${ }^{2-4}$ However, we have found evidence of prolonged psychophysical dark adaptation in several patients with RP. Of the nine patients and three X-linked recessive carriers who were tested five patients and two carriers showed a delayed recovery of sensitivity, ranging from mild to severe. The prolonged dark adaptation was not restricted to a particular retinal region but was found at the two retinal locations that

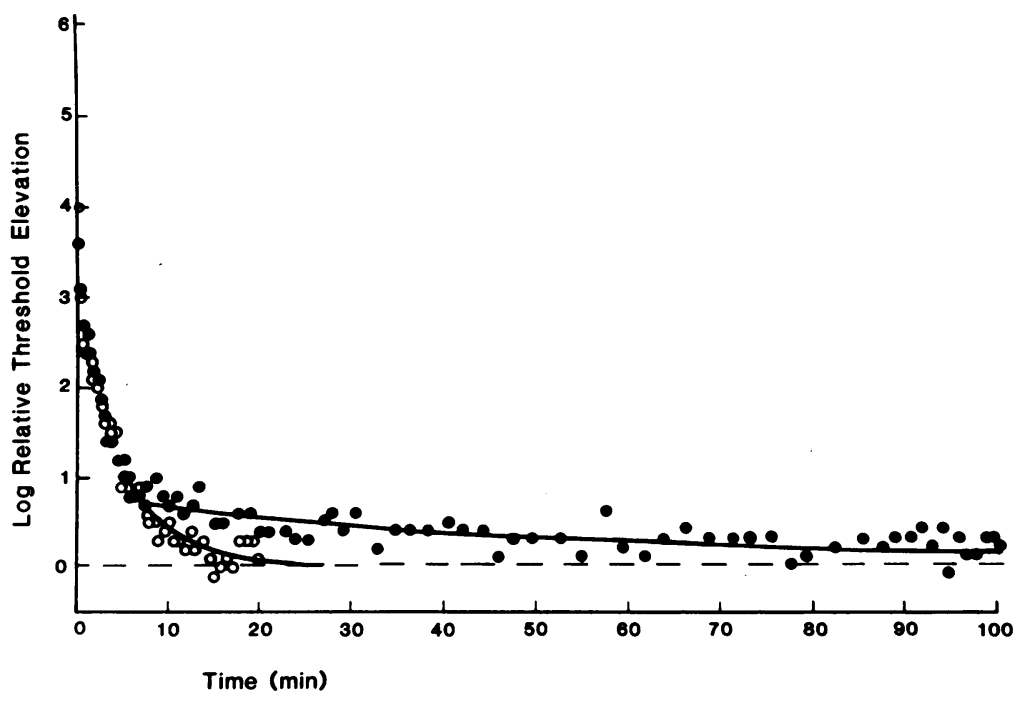

Fig. 11 Thresholds for the middle-wavelength test flash during dark adaptation, following a 30 s exposure to the weak bleach, for patient I (filled circles) at a retinal ecceniricity of $45^{\circ}$ and one normal subject (open circles) at a retinal eccentricity of $20^{\circ}$. The solid line through the open circles represents the expected threshold values based on the regeneration of rhodopsin, with a time constant of 6 min (see text for details). The solid line through the filled circles represents the best-fitting exponential for patient 1 , with a time constant of 52.7 min. Curve fitting was begun at the point where thresholds diverged from normal (other details as in Fig. 2). 


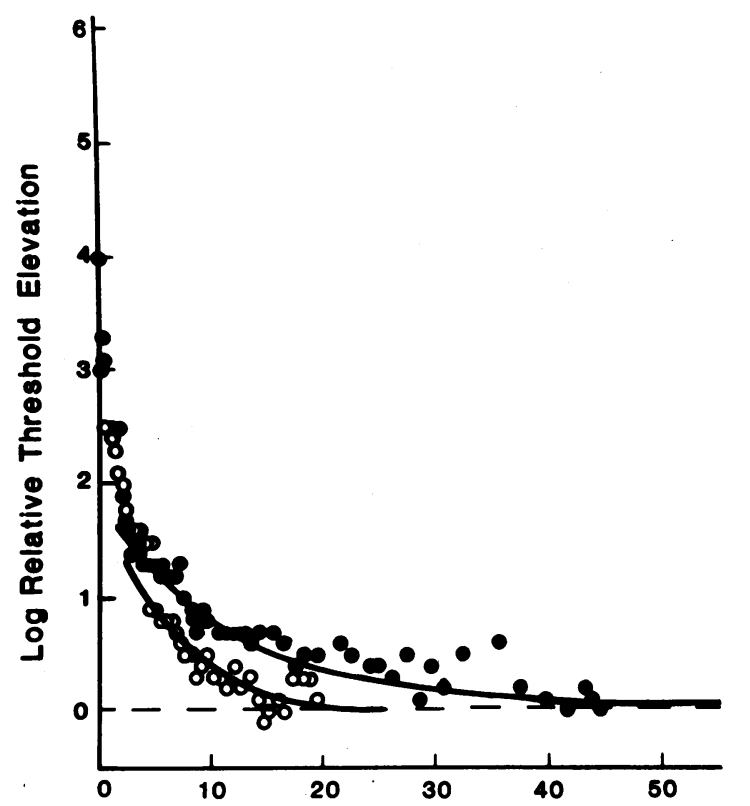

Time (min)

Fig. 12 Thresholds during dark adaptation following the weak bleach for patient 3 (filled circles) at a retinal eccentricity of $20^{\circ}$, with a time constant of 13 min (other details as in Figs. 2 and II).

were tested in each patient. Furthermore, the prolongation appears to be unrelated to the degree of rod sensitivity loss, since retinal regions that had nearly normal absolute thresholds sometimes exhibited a marked prolongation.

One of the primary differences between our procedure and that of earlier investigators ${ }^{2-4}$ is that we measured the prebleach dark-adapted sensitivity of these patients to establish a baseline to which the patients' sensitivity should return. ${ }^{5}$ Because the dark adaptation was so prolonged in several of our RP patients, without such a baseline it might have been erroneously concluded that the bleaching recovery of these patients had ended at the normal time.

In four of the dark adaptation curves reported (Figs. 2, 4, 7, 10) the thresholds failed to reach the prebleach level before the testing session was terminated owing to patient considerations. It is unlikely that the failure to reach the prebleach value was the result of an error in measuring the prebleach threshold. First, we retested the absolute thresholds of three patients (nos. 1, 3, and 5) in subsequent sessions and found that the values differed at most by only a few tenths of a log unit between sessions. This variability is comparable to that of our normal observers and is not large enough to account for our results. Secondly, prolonged dark adaptation curves with similar time constants were observed at both of the retinal locations that were tested in each patient. Such consistency in results would be unlikely to occur if the prolonged dark adaptation were simply the result of changes in fixation. Thirdly, as shown in Figs. 11 and 12, rod thresholds ultimately reached their prebleach value if dark adaptation was measured after a weaker bleach. Therefore it is most likely that the failure of the rod thresholds to reach their prebleach value in Figs. 2, 4, 7, and 10 is the result of a prolonged rod bleaching recovery.

The prolonged time course of rod dark adaptation observed in these RP patients and carriers is most probably due to an abnormality in photopigment bleaching and/or regeneration kinetics. However, since the relationship between visual threshold and the underlying photochemical events in the normal eye is uncertain at present, ${ }^{510}$ the specific mechanism responsible for the prolonged rod dark adaptation cannot be specified. Nevertheless, it is of interest that the prolongation of dark adaptation appears to be limited to the later phase of rod recovery. This is shown most clearly by one $\mathrm{X}$-linked recessive carrier (patient 2) following the moderate bleach and by patients 1 and 3 following the weak bleach.

For patient 2 the thresholds between 7 and 13 minutes after bleach (Fig. 4) follow the normal course of bleaching recovery. At $13 \mathrm{~min}$, however, dark adaptation slows substantially and then proceeds with a delayed time course. (Presumably a similar normal recovery of rod sensitivity early in dark adaptation would be observed in the other RP patients if cones did not prevent the measurement of rod sensitivity.) This biphasic recovery of rod sensitivity may be due to a regeneration of rhodopsin that proceeds at two different rates, similar to that reported by Alpern and Krantz in uveal disease. ${ }^{14}$ Alternatively, the flattening of the rod recovery curve might reflect the slow removal of a bleaching photoproduct that desensitises the rods. "'

For RP patients 1 and 3 the initial portion of the dark adaptation curve following the weak bleach is normal (Figs. 11 and 12), whereas the later phase is abnormally slow. The early portion of rod dark adaptation under these conditions has been shown to be unrelated to the concentration of bleached rhodopsin, and has been variously attributed to 'neural' factors ${ }^{15}$ or to the presence of putative bleaching photoproducts such as 'X-opsin' ${ }^{\prime 1}$ or ' $\mathrm{S}_{1}{ }^{11}$ "' The later phase of dark adaptation following a weak bleach has generally been attributed to the regeneration of bleached rhodopsin." It is this later phase of dark adaptation following the weak bleach that is abnormal in our RP patients 1 and 3 .

These results suggest that there is an impairment in the processes underlying rod dark adaptation in these 
patients and carriers that may be related to the regeneration of rhodopsin. Previous studies of rhodopsin regeneration in RP patients, by the technique of reflection densitometry, have shown that the regeneration rate is within normal limits. ${ }^{1718}$ However, only a few patients have been studied, and it is possible that results would have been different in other patients. Conversely, the prolonged rod dark adaptation may be due to factors other than delayed rhodopsin regeneration, such as the slow removal of bleaching by products that desensitise the rods. ${ }^{510}$ Further research is needed to determine the basis for the prolonged rod dark adaptation in these RP patients and carriers.

This work was supported in part by NIH Training Grant EY07038, NIH Core Grant EY01792, and a grant from the National Retinitis Pigmentosa Foundation.

We thank Dr R. Massof for the generous loan of the auxiliary fixation device, and Dr M. F. Goldberg, Dr L. Hutman, and Ms M. Gere for helpful comments on the manuscript.

\section{References}

1 Merin S, Aucrbach E. Retinitis pigmentosa. Surv Ophthalmol 1976; 20: 303-46.

2 Mandelbaum J. Dark adaptation: some physiologic and clinical considcrations. Arch Ophthalmol 1941; 26: 203-39.

3 Zcavin $\mathrm{BH}$, Wald $\mathrm{G}$. Rod and cone vision in retinitis pigmentosa. Am J Ophthalmol 1956; 42: 253-68.
4 Goodman G, Gunkel RD. Familial clectroretinographic and adaptometric studies in retinitis pigmentosa. Am J Ophthalmol 1958; 46: 142-72.

5 Pugh EN Jr. Rushton's paradox: rod dark adaptation after flash photolysis. J Physiol (Lond) 1975; 248: 413-31.

6 Rushton WAH. Rhodopsin measurement and dark adaptation in a subject deficient in cone vision. J Physiol (Lond) 1961; 156: 193-205.

7 Alpern M. Rhodopsin kinetics in the human cyc. J Physiol (Lond) 1971; 217: 447-71.

8 Dowling JE. Neural and photochemical mechanisms of visual adaptation in the rat. J Gen Physiol 1963; 46: 1287-301.

9 Rushton WAH, Powell DS. The rhodopsin content and the visual threshold of human rods. Vision Res 1972; 12: 1073-81.

10 Lamb TD. The involvement of rod photoreceptors in dark adaptation. Vision Res 1981; 21: 1773-82.

11 Fishman GA, Anderson RJ, Stinson L, Haque A. Driving performance of retinitis pigmentosa patients. BrJ Ophthalmol 1981; 65: 122-6.

12 Massof RW, Finkelstein D. Two forms of autosomal dominant primary retinitis pigmentosa. Doc Ophthalmol 1981;51:289-346.

13 Hecht S, Haig C, Wald G. The dark adaptation of retinal fields of different size and location. J Gen Physiol 1935; 19: 321-37.

14 Alpern M, Krantz DH. Visual pigment kinctics in abnormalitics of the uvea-retinal epithelium interface in man. Invest Ophthalmol Visual Sci 1981; 20: 183-203.

15 Dowling JE, Ripps H. Adaptation in skatc photoreceptors. J Gen Physiol 1972; 60: 698-719.

16 Rushton WAH, Powcll DS. The carly phase of dark adaptation. Vision Res 1972; 12: 1083-93.

17 Highman VN, Wealc RA. Rhodopsin density and visual threshold in retinitis pigmentosa. Am J Ophthalmol 1973; 75: 822-32.

18 Ripps H, Brin KP, Wcalc RA. Rhodopsin and visual threshold in retinitis pigmentosa. Invest Ophthalmol Visual Sci 1978; 17: 735-45. 